\title{
Time-Resolved Observations of Liquid-Liquid Phase Separation at the Nanoscale using in situ Liquid Transmission Electron Microscopy
}

Hortense Le Ferrand ${ }^{1}$, Martial Duchamp ${ }^{2}$, Bartosz Gabryelczyk ${ }^{1}$, Hao Cai ${ }^{1}$, and Ali Miserez ${ }^{1,3 *}$

${ }^{1}$ Biological and Biomimetic Materials Laboratory and Center for Biomimetic Sensor Science, School of Materials Science and Engineering, Nanyang Technological University (NTU), 50 Nanyang Avenue, Singapore 637553.

${ }^{2}$ Laboratory for in situ \& operando Electron Nanoscopy, School of Materials Science and Engineering, Nanyang Technological University (NTU), 50 Nanyang Avenue, Singapore 637371.

${ }^{3}$ School of Biological Sciences, Nanyang Technological University (NTU), 60 Nanyang Drive, Singapore 637551.

*Corresponding author: ali.miserez@ntu.edu.sg 


\section{Supplementary Material}

\section{Supplementary Information}

Protein concentration in coacervate microdroplets. A $10 \mathrm{mg} / \mathrm{mL}$ protein solution in $10 \mathrm{mM}$ acetic acid was placed in high coacervation buffer $(\mathrm{pH} 6.5,[\mathrm{NaCl}]=1 \mathrm{M})$. To determine the concentration, the coacervate microdroplets formed were crosslinked using 4-methyl catechol (4MC, Sigma Aldrich, India) at a concentration of $64 \mathrm{mg} / \mathrm{mL}$ and a volume ratio protein: 4MC of 1:1. $\mathrm{NaIO}_{4}$ (Aldrich, India) at a concentration of $58 \mathrm{mg} / \mathrm{mL}$ was also added with the same volume and thoroughly mixed by pipetting. After overnight crosslinking at room temperature, the colloidal solution turned brown and was centrifuged at $150 \mathrm{rpm}$ for 10 minutes and the supernatant exchanged with miliQ water three times to remove unreacted salt. After that, a small volume of crosslinked coacervate microdroplets was placed in glucose (BBI Life Sciences) solutions at different $w t \%$ in glucose. The absorbance of the solution placed in a disposable plastic cuvette was recorded at $274 \mathrm{~nm}$ as a function of time using a spectrophotometer (Nanodrop 2000c, Thermo Fisher Scientific) under the UV-Vis mode. The measurement was repeated 3 times with independent initial solutions.

The concentration of protein inside the droplet was estimated using the rule of mixture:

$$
\rho_{\text {droplet }}=\varphi_{\text {prot }} \cdot \rho_{\text {prot }}+\left(1-\varphi_{\text {prot }}\right) \cdot \rho_{w}
$$

where $\rho_{\text {droplet }}, \rho_{\text {prot }}$ and $\rho_{w}$ are the measured densities of the microdroplets, proteins, and water, respectively, and $\varphi_{\text {prot }}$ the volume fraction of protein inside the microdroplet.

Re-ordering the terms and replacing by the measured values gives:

$$
\varphi_{\text {prot }}=\frac{\rho_{\text {droplet }^{-1}}}{\rho_{\text {prot }^{-1}}}=68.4 v_{\text {ol }} \%=90 \mathrm{wt} \%=900 \mathrm{mg} / \mathrm{mL}
$$

This protein concentration in the coacervate microdroplet is close to the concentration measured or predicted by others (Supplementary Ref. 1).

Zeta Potential. The Zetasizer Nano SZ-100 (Horiba) was used to measure the zeta potential of protein solutions at $\mathrm{pH}$ from 2 to 6.5 , without the presence of $\mathrm{NaCl}$. A total volume of $250 \mu \mathrm{L}$ of the protein solution at $10 \mathrm{mg} / \mathrm{mL}$ of protein was placed into a disposable plastic cell with carbon electrodes. 3 measurements of $30 \mathrm{~s}$ each were performed at room temperature and analyzed using the Smoluchowski approximation. 


\section{Supplementary Figures}

\begin{tabular}{|c|c|c|c|c|c|c|c|c|c|}
\hline \multicolumn{8}{|c|}{ Number of amino acids } & Ala-rich & GHGPY \\
\hline 0 & 25 & 50 & 75 & 100 & 125 & 150 & 175 & Hid & \\
\hline
\end{tabular}

Supplementary Figure 1 | Primary structure of HBP-2, reproduced from Ref. [23].
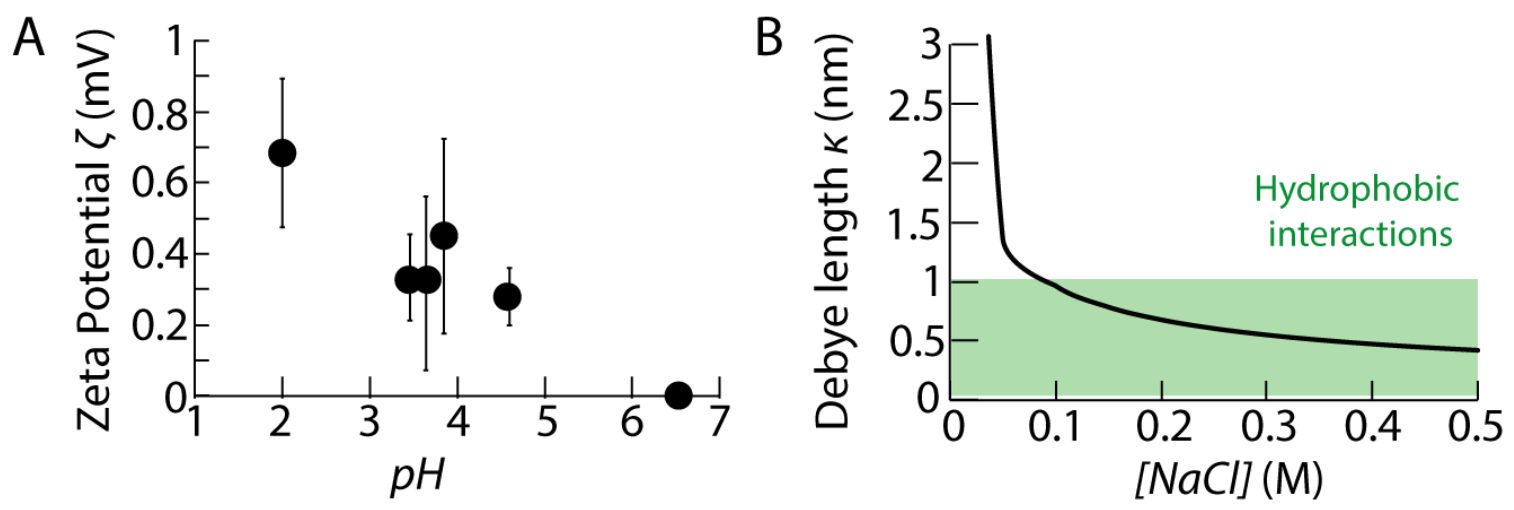

Supplementary Figure 2 | (A) Zeta potential of HBP-2 measured at a protein concentration of $10 \mathrm{mg} / \mathrm{mL}$ at different $\mathrm{pH}$, without addition of salt. (B) Calculation of the Debye length $\kappa$ as a function of $[\mathrm{NaCl}]$ concentration using the equation $\kappa=\frac{1}{\sqrt{4 \pi l_{B} c_{i o n}}}$, where $c_{\text {ion }}$ is the salt concentration, $l_{B}$ the Bjerrum length $l_{B}=\frac{q^{2}}{\varepsilon k_{B} T}, k_{B}$ the Boltzmann constant, $T$ the temperature, $q$ the ionic charge, and $\varepsilon$ the dielectric constant of the medium. 

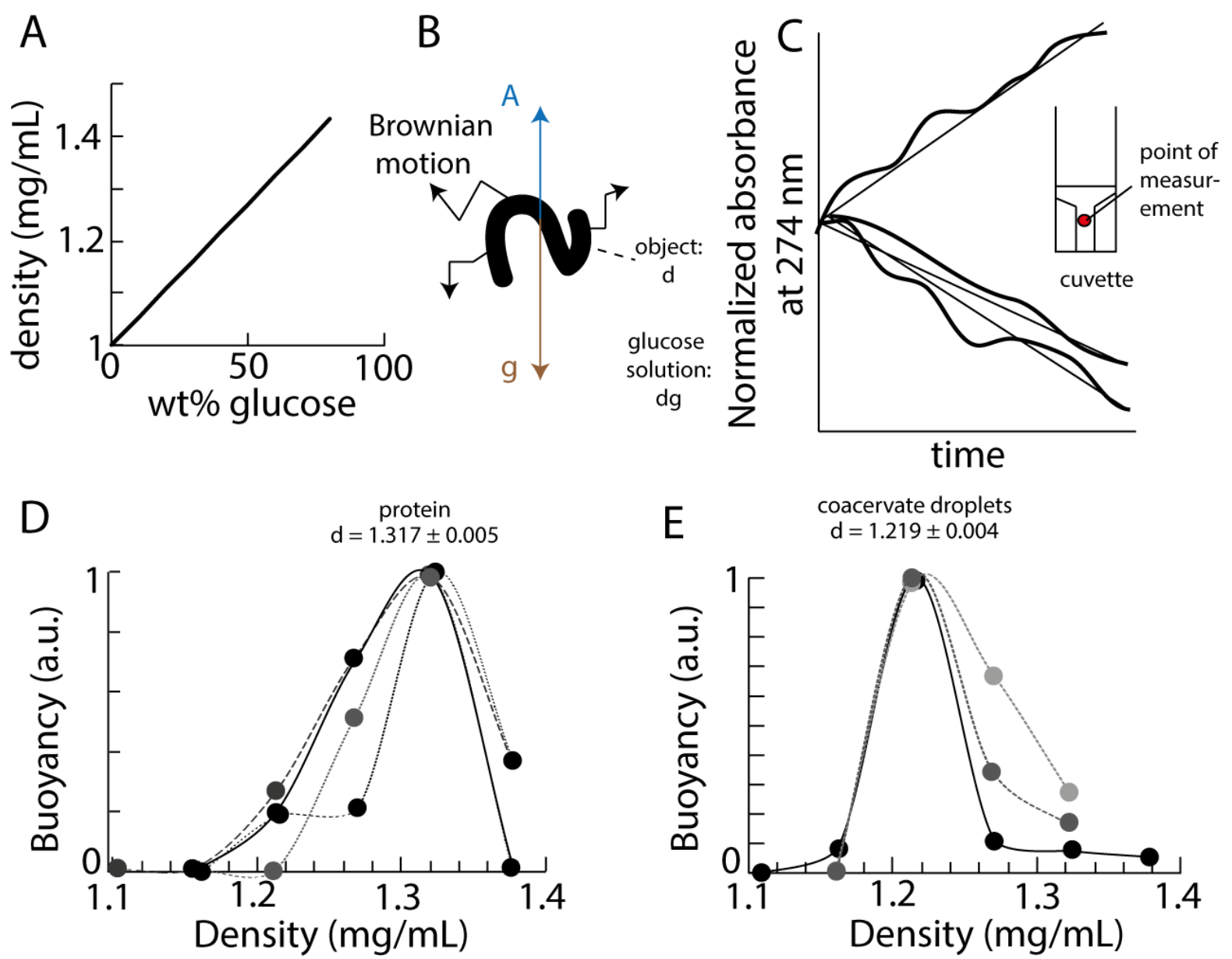

Supplementary Figure 3 | Determination of the density of coacervate microdroplets by matching index in glucose solutions. (A) Increase in density as a function of glucose wt.\% in glucose solutions. (B) Principle of the method: cross-linked protein microdroplets are suspended into a glucose solution of density dg. The proteins are submitted to gravity, Brownian motion, and Archimedes force. (C) Principle of buoyancy measurements (a.u.): the absorbance at $274 \mathrm{~nm}$ in the middle of the cuvette is measured as a function of time. At the matching density between the protein solution and the glucose solution, the proteins are submitted only to Brownian motion and the absorbance at the middle of the cuvette increased with time due to mixing effect. Under nonmatching density conditions, the absorbance decreased as the protein settled to the bottom of the cuvette or was lifted to the air/surface interface. (D, E) Normalized buoyancy of (D) cross-linked unfolded proteins, and (E) coacervate microdroplets as a function of the density of glucose solutions. A normalized buoyancy of 1 indicates a matching density between the glucose solution and the object. 

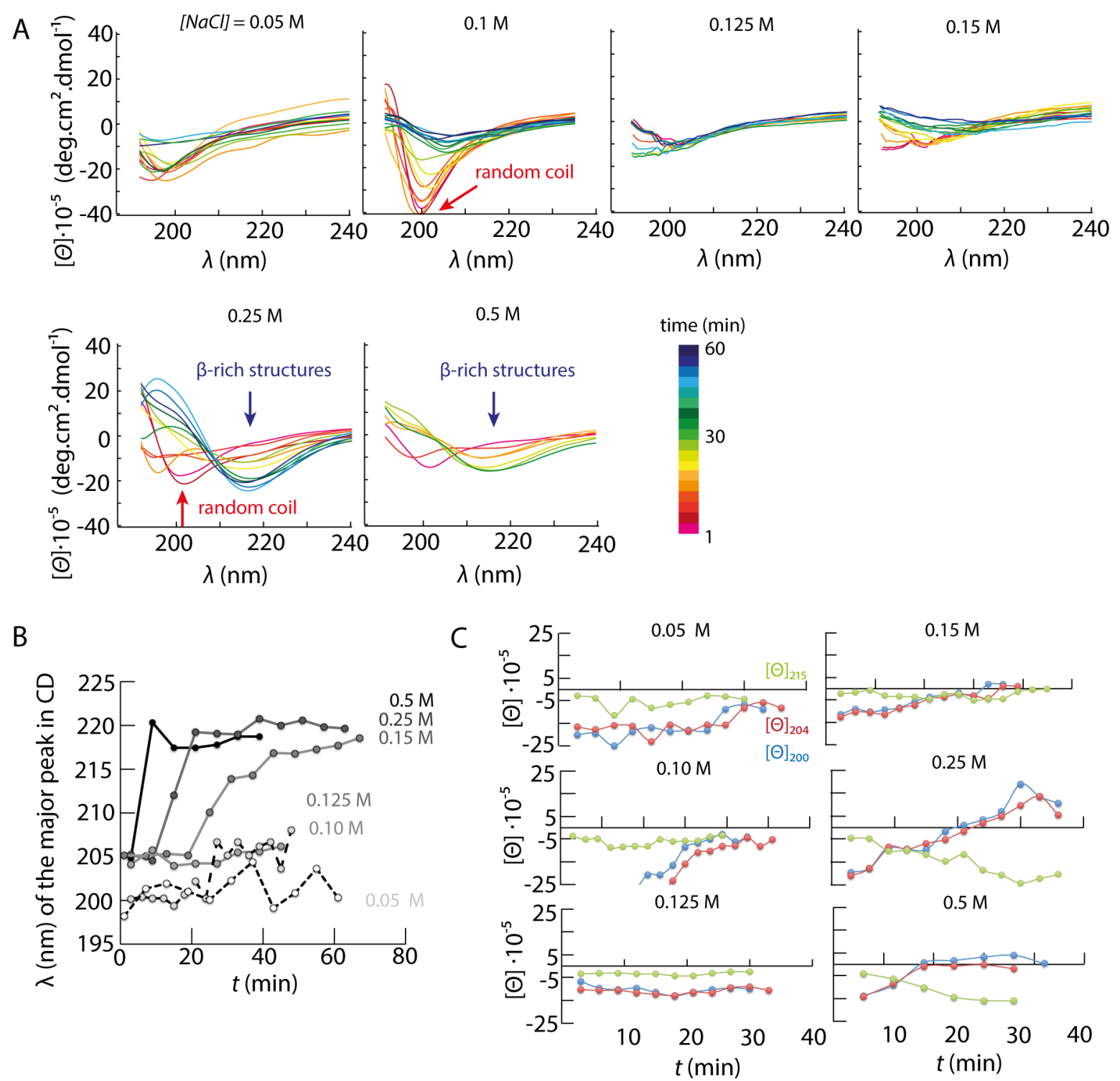

Supplementary Figure 4 | (A) Circular dichroism spectra representing the ellipticity $\theta$ as a function of the wavelength $\lambda$ with color highlighting their time dependency. From left to right the spectra were recorded in the same conditions of $\mathrm{pH}=5.5$ and initial protein concentration $C_{0}$, but with an increasing salt concentration of $[\mathrm{NaCl}]$. (B) Wavelength $\lambda$ of the major peak (minimum) in the CD spectra as a function of time and salt concentration. (C) Time evolution of the ellipticity $\theta$ at $\lambda=200 \mathrm{~nm}$ (blue), $204 \mathrm{~nm}$ (red) and $215 \mathrm{~nm}$ (green). 

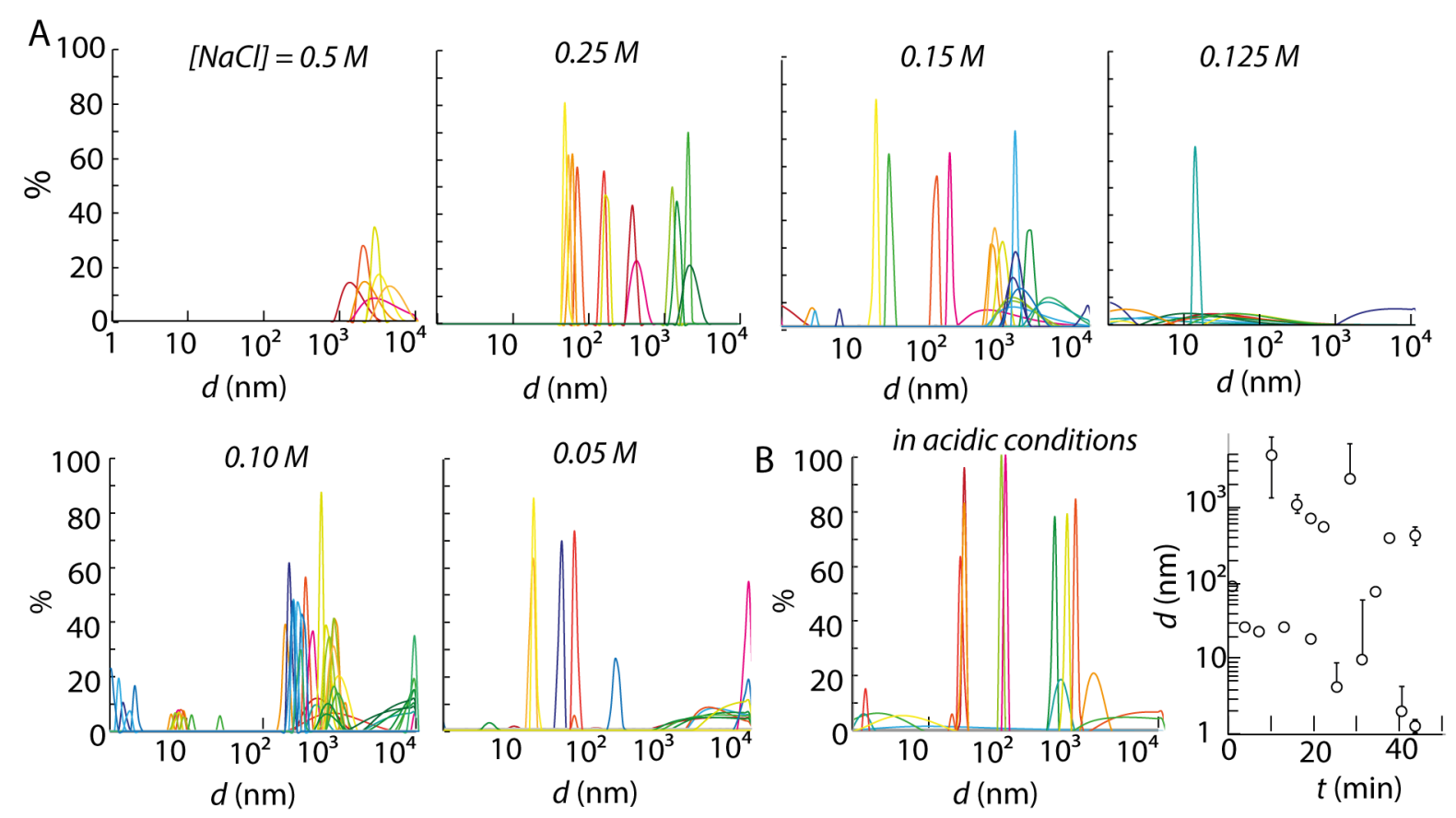

Supplementary Figure 5 | (A) Size distributions of protein nanoclusters measured by dynamic light scattering at decreasing salt concentration at $\mathrm{pH}$ 5.5. (B) Size distribution and diameter of the nanoclusters formed as a function of time for the protein acidic solution. 

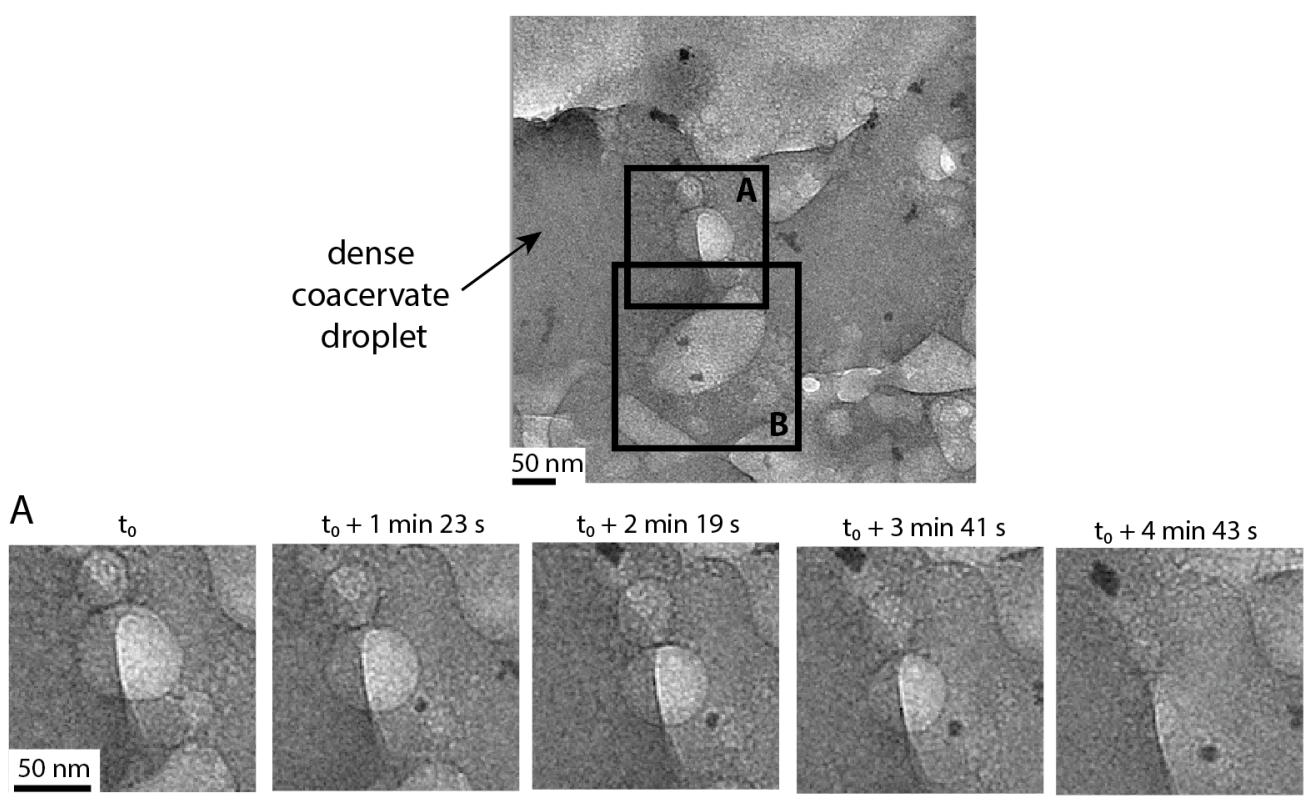

$t_{0}+5 \min 27 s$

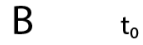

$t_{0}+1 \min 23 s$

$t_{0}+2 \min 19 s$

$t_{0}+3 \min 41 \mathrm{~s}$
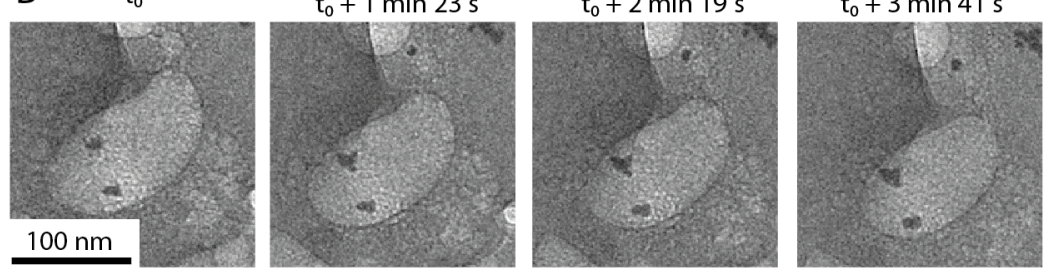

$t_{0}+4 \min 43 s$

$t_{0}+5 \min 27 s$
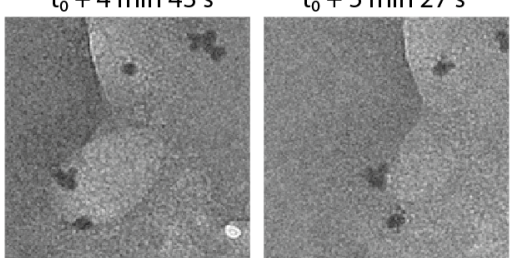

Supplementary Figure 6 | (A, B) Time lapse high resolution images obtained by in situ liquid TEM of HBP-2 protein at a salt concentration $[\mathrm{NaCl}]=0.15 \mathrm{M}$, showing the shrinking of vesiclelike features near the vicinity of the dense coacervate microdroplet as indicated in the first micrograph. 

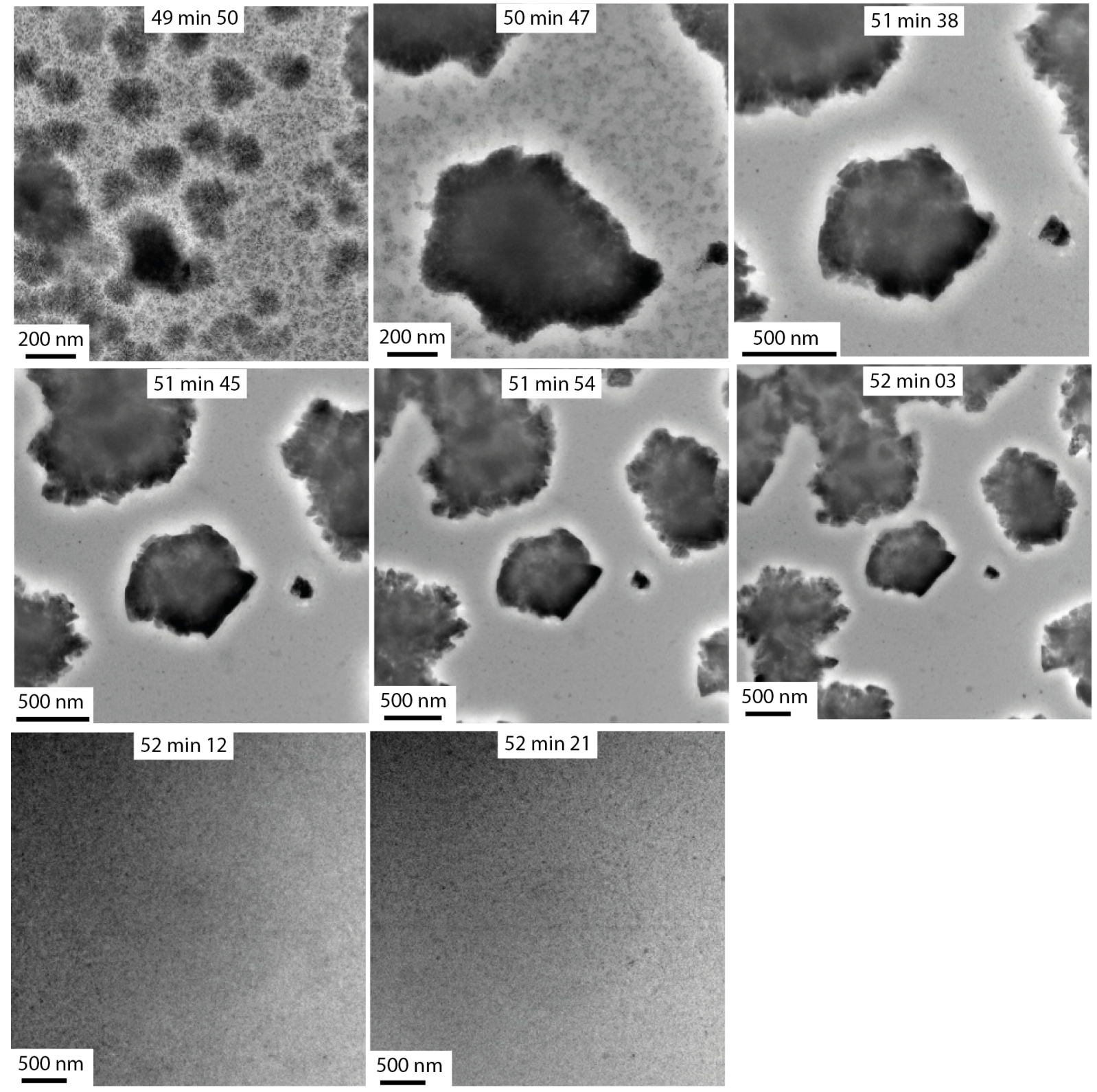

Supplementary Figure 7 | Time-lapse high resolution images obtained by in situ liquid TEM of HBP-2 protein at a salt concentration $[\mathrm{NaCl}]=0.10 \mathrm{M}$, showing the assembly, growth, and redissolution of protein nanoclusters. The grey contrast after re-dissolution highlights the fact that the proteins do not recover their initial random coil configuration but transit into another intermediate state. 


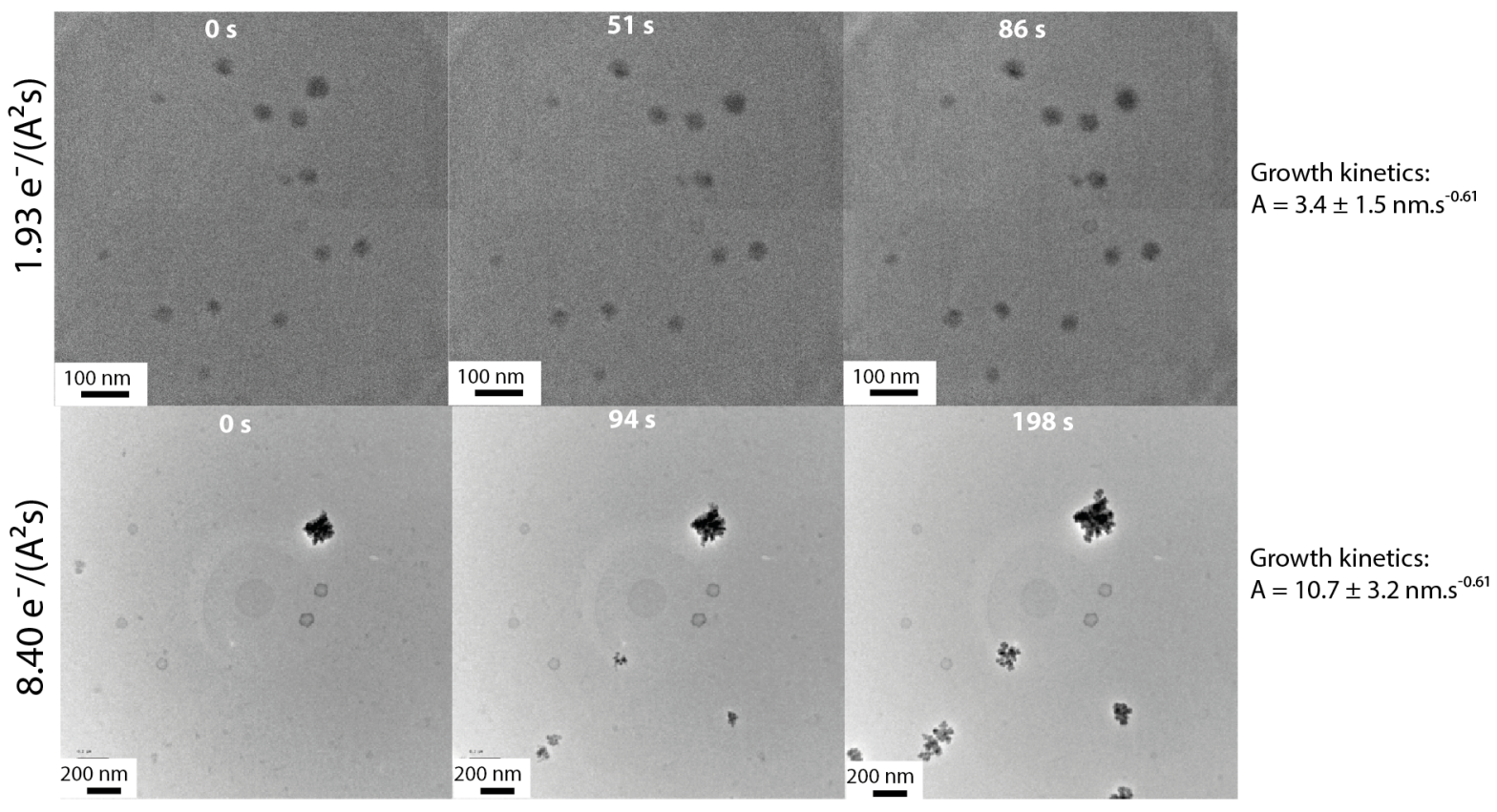

Supplementary Figure 8 | Time-lapse micrographs obtained by in situ liquid TEM of HBP-2 protein at a salt concentration $[\mathrm{NaCl}]=0.137 \mathrm{M}$, and with increasing electron dose rate indicated on the right, showing an increased growth rate $A$ of protein nanoclusters. The values of $A$ were obtained using $d=A \cdot t^{0.61}+B$ and averaged over 4 nanoclusters.
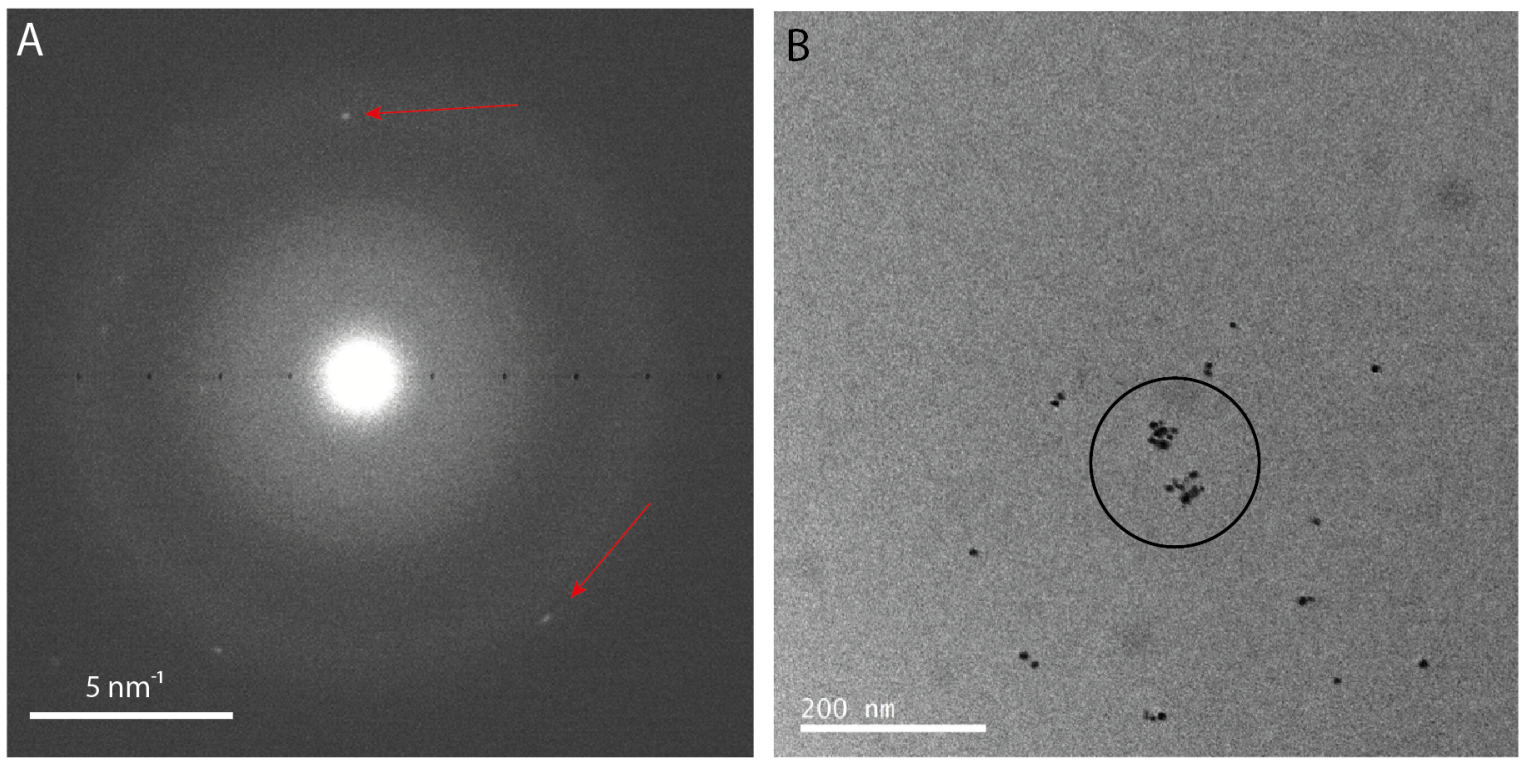

Supplementary Figure 9 | (A) Diffraction by selected area diffraction carried out in liquid TEM at $[\mathrm{NaCl}]=0.10 \mathrm{M}$. The red arrows indicate diffraction spots that correspond to diffraction of crystalline $\mathrm{NaCl}$. (B) Corresponding bright field micrograph with the area selected for diffraction highlighted with the black circle. 


\section{Supplementary Tables}

Supplementary Table 1 | Fitting parameters of the ellipticity $\theta$ at $\lambda=215 \mathrm{~nm}$ as a function of time using $\theta(t)=A \cdot e^{\frac{-t}{\tau}}+B$.

\begin{tabular}{lllll} 
Salt (M) & $\mathbf{A}$ & $\mathbf{B}$ & $\mathbf{1} / \boldsymbol{\tau}$ & $\boldsymbol{R}^{\mathbf{2}}$ \\
\hline 0.5 & 6.72 & -7.61 & 85.8 & 0.88 \\
0.25 & 12.53 & -13.04 & 36.11 & 0.96 \\
0.15 & 3.27 & -5.53 & 7.34 & 0.85 \\
0.10 & 29.98 & -0.05 & 20.83 & 0.85 \\
0.05 & 1.215 & -2.42 & 0.01 & 0.95
\end{tabular}

Supplementary Table 2 | Fitting parameters of the normalized density of microdroplets curves using $J \cdot\left(t+t_{\text {lag }}\right)$. For $[\mathrm{NaCl}] \leq 0.125 \mathrm{M}$, no microdroplets were observed during the time of the measurement.

\begin{tabular}{llll} 
Salt $(\mathbf{M})$ & $\mathbf{J}\left(\mathbf{m i n}^{-\mathbf{1}}\right)$ & $\mathbf{t}_{\text {lag }}(\mathbf{m i n})$ & $\boldsymbol{R}^{\mathbf{2}}$ \\
\hline 1 & 16.23 & 0.55 & 0.74 \\
0.5 & 10.04 & 2.06 & 0.89 \\
0.25 & 0.19 & 3.46 & 0.89 \\
0.15 & 0.11 & 17.83 & 0.98
\end{tabular}

Table 3 | Fitting parameters of the protein nanocluster growth (kinetic factor A) obtained from the fitting of the evolution of the nanocluster sizes with time using the equation: $d=A \cdot t^{n}+B$, with $n=0.61$.

\begin{tabular}{lllll} 
Salt $(\mathbf{M})$ & Color curve & $\mathbf{A}\left(\mathbf{n m} \cdot \mathbf{s}^{-\mathbf{0 . 6 1}}\right)$ & $\boldsymbol{B}$ & $\boldsymbol{R}^{\mathbf{2}}$ \\
\hline 0.1 & Black & 119.29 & 91.7 & 0.95 \\
0.1 & Dark grey & 173.04 & 55.2 & 0.93 \\
0.1 & Medium grey & 95.72 & 88.5 & 0.96 \\
0.1 & Light grey & 51.93 & 162.3 & 0.96 \\
0.125 & Black & 40.67 & 46 & 0.99 \\
0.125 & Dark grey & 33.14 & 71.47 & 0.99 \\
0.125 & Light grey & 25.81 & 52.56 & 0.97
\end{tabular}




\section{Supplementary Movies}

Supplementary Movie 1 | Movie obtained by in situ liquid TEM of HBP-2 (pH 5.5; [NaCl] = $0.15 \mathrm{M})$.

Supplementary Movie 2 | Movie obtained by in situ liquid TEM of HBP-2 (pH 5.5; [NaCl] = $0.125 \mathrm{M})$.

Supplementary Movies 3 \& 4 | Movies obtained by in situ liquid TEM of HBP-2 (pH 5.5; [NaCl] $=0.10 \mathrm{M})$.

\section{Supplementary Reference}

1 Dignon, G.L., Zheng, W., Best, R.B., Kim, Y.C. \& Mittal, Jeetain. Relation between single-molecule properties and phase behaviour of intrinsically disordered proteins. PNAS 115, 9929-9934 (2018). 